\title{
TRATAMENTO TÉRMICO EM MATERIAIS TÊXTEIS COMPOSTOS DE FIBRAS SINTÉTICAS.
}

\author{
J.B.GIORDANO ${ }^{1}$ \\ ${ }^{1}$ Faculdade de Tecnologia de Americana. Departamento de Têxtil \\ E-mail para contato: jbgiordano@uol.com.br
}

\begin{abstract}
RESUMO: O tratamento térmico aplicado em materiais compostos de fibras sintéticas e é denominado na indústria têxtil como termofixação. Este processo tem a finalidade conferir estabilidade dimensional ao tecido, porém pode alterar outras propriedades, como grau de cristalinidade, afinidade tintorial, alongamento e ruptura. O objetivo deste trabalho é verificar a ação da temperatura de tratamento nas características dos tecidos sintéticos. Neste trabalho forma utilizados tecidos de poliéster e de poliamida. Amostras foram submetidas a temperaturas e tempos e em seguidas foram avaliados suas propriedades mecânicas, alterações dimensionais e afinidade tintorial. Observou-se que nas temperaturas de $180^{\circ} \mathrm{C}$ e $140^{\circ}$ os tecidos de poliéster e poliamida obtiveram boa estabilidade. A resistência a tração e alongamento tende a aumentar até nestas temperaturas, acima destes valores estas propriedades diminuem, pois começa degradação do material. Afinidade tintorial diminui com o aumento da temperatura, indicando aumento da cristalinidade dos materiais. Este tratamento deve ser feito nestas temperaturas na fase inicial do beneficiamento destes materiais para se obter uniformidade nos tecidos, tanto na suas dimensões (sem encolhimentos futuros) e reprodução da cor do tingimento para ocorres isso, os processos posteriores devem ser executados em temperaturas inferiores ao tratamento térmico realizado preliminarmente.
\end{abstract}

\section{INTRODUÇÃO:}

O processo de termofixação é baseado no aquecimento dentro de uma faixa específica de temperatura cujo limite superior é a temperatura de fusão e o limite inferior é a temperatura de transição vítrea da fibra (necessária para quebrar as ligações secundárias). (Vasquez e Sakellarios, 1980)

Os principais objetivos obtidos pelo processo de termofixação são: (Menachen e Preston

- Homogeneização da estrutura da fibra;

- Eliminação de tensão interna da fibra, resultando em redução do encolhimento durante processos de beneficiamento e uso final; 


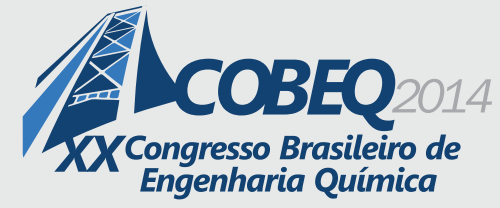

- Aumento da estabilidade dimensional;

Os parâmetros mais importantes na temofixação são temperatura, tempo e a tensão mecânica aplicada ao tecido.

As mudanças causadas pela termofixação não são apenas mecânicas, mas também nas propriedades de tingimento das fibras sintéticas. A temperatura determina as propriedades de tingimento como segue: Com o aumento da temperatura (no caso do poliéster, acima de $200{ }^{\circ} \mathrm{C}$ ) a proporção de regiões cristalinas diminui porque os cristalitos instáveis fundem e a massa polimérica recristaliza parcialmente. Quanto maior a proporção de regiões não cristalinas depois da termofixação, que também é influenciada pela taxa de resfriamento (resfriamento rápido reduz a recristalização), melhores serão as propriedades de tingimento posterior. (Odian, 1991)

Os substratos possuem tensões adquiridas durante seus processamentos. Estas forças são liberadas nas operações úmidas do beneficiamento manifestando-se na forma de encolhimento ou alongamento (modificações das dimensões).

Este encolhimento torna-se mais acentuado se os banhos forem aquecidos e para evitar estas distorções deve-se fixar o substrato previamente em temperaturas superiores às das operações que posteriormente serão realizadas.

Observa-se que os substratos compostos de fibras sintéticas são os que mais sofrem problemas de estabilidade dimensional. (Bernard, 1983)

Existem quatro métodos para a pré-fixação dos substratos têxteis, são eles: (Billmeyr,

a) Hidrofixação: Neste método o substrato é mergulhado num banho aquecido onde permanece o tempo necessário para sua fixação. Por este método podem ser beneficiadas fibras, fios, filamentos, tecidos planos (feitos no tear) e de malha e confeccionados.

b) Fixação por convecção ou fluxo de ar (Termofixação): Neste método o substrato é fixado por meio de massas de ar aquecidas que circulam por convecção. Por este motivo, leva o nome de fixação por convecção. Este método é empregado para o beneficiamento de fios, filamentos, tecidos planos e de malha e também para confeccionados.

c) Fixação por vaporização: Neste método de fixação o substrato recebe uma carga de vapor saturado. Esta forma é empregada para a fixação de todas as formas de apresentação de substrato.

d) Fixação por contato: Aqui o substrato recebe a fixação em contato com uma placa aquecida. Emprega-se este método para tecidos de cala, de malha e confeccionados. 
Temperatura mínima de fixação: Baixa estabilidade dimensional, toque macio e volumoso e maior sensibilidade à formação de rugas.

Temperatura elevada de fixação: Elevada estabilidade dimensional, toque duro e fino e menor sensibilidade à formação de rugas

\subsection{Alteração Dimensional de tecidos}

$\mathrm{Na}$ produção dos tecidos e nos seus diferentes processos de beneficiamento, surgem alterações dimensionais devido às ações mecânicas, térmicas e químicas que, na maioria dos casos, se manifestam posteriormente, por encurtamento na direção longitudinal. Este fenômeno é geralmente referido como encolhimento. A quantidade que encolhe um tecido depende principalmente do material, das condições de produção, do ambiente que o tecido é beneficiado (água, vapor, calor, seco, etc.), a temperatura e os esforços mecânicos aos quais ele é submetido.

O mecanismo do encolhimento pode ser explicado pelo alívio de tensões internas introduzidas nos fios durante os processos de fiação e tecelagem. Pela atuação de água, ar quente ou vapor, as tensões podem ser aliviadas. Melhorando a estabilidade das dimensões, e o tempo necessário para isto vai depender do tipo da fibra, do tipo e condições de tratamentos aplicados. (Vasquez e Sakellarios, 1980).

\section{EXPERIMENTAL:}

\section{1. Materiais e equipamentos:}

\section{Materiais:}

- Tecidos de poliéster, tecidos de poliamida, régua milimetrada, corantes dispersos, corantes ácidos, ácido acético, sabão neutro, água e vidraria comum de laboratório.

\section{Equipamentos:}

- Balança de precisão $(0,001 \mathrm{~g})$, estufa com controle de tempo e temperatura, dinamômetro, aparelho de tingimento de poliéster e de poliamida, máquina de lavar roupa caseira.

\subsection{Metodologia:}

\section{$\underline{\text { Tecidos de poliéster: }}$}

- Amostras de tecidos de poliéster foram submetidas a temperaturas de 100, 120, 140, 160, 180 e $200^{\circ} \mathrm{C}$ por $2 \mathrm{~min}$.

- Em seguida, foram feitas nestas amostras medidas de gramatura de tecido $\left(\mathrm{g} / \mathrm{m}^{2}\right)$, porcentagem de encolhimento no sentido trama e urdume, ensaio de resistência a tração e porcentagem de alongamento no momento de ruptura da amostra, Tingimento destas amostras utilizando corante 
disperso (tricromia de amarelo, azul e vermelho) e ensaio de estabilidade dimensional (lavagens sucessivas a temperatura de $25,60,80$ e $98^{\circ} \mathrm{C}^{\circ}$ utilizando $2 \mathrm{~g} / \mathrm{l}$ sabão neutro)

Tecidos de poliamida:

- Amostras de tecidos de poliamida foram submetidas a temperaturas de 100, 120, 140, 160, 180 e $200^{\circ} \mathrm{C}$ por $2 \mathrm{~min}$.

- Em seguida, foram feitas nestas amostras medidas de gramatura de tecido, porcentagem de encolhimento no sentido trama e urdume, ensaio de resistência a tração e porcentagem de alongamento no momento de ruptura da amostra, Tingimento destas amostras utilizando corante ácido (tricromia de amarelo, azul e vermelho) e ensaio de estabilidade dimensional (lavagens sucessivas a temperatura de $25,60,80$ e $98^{\circ} \mathrm{C}^{\circ}$ utilizando $2 \mathrm{~g} / \mathrm{l}$ sabão neutro).

\section{RESUTADOS E DISCUSSÕES:}

\subsection{Tecidos de poliéster:}

Tabela 1 - Resultados das propriedades dos tecidos de poliéster tratados termicamente nas temperaturas de $100,120,140,160,180$ e $200^{\circ} \mathrm{C}$ e de tecidos não tratados $\left(25^{\circ} \mathrm{C}\right)$

\begin{tabular}{|c|c|c|c|c|c|c|c|}
\cline { 2 - 8 } \multicolumn{2}{c|}{} & \multicolumn{7}{c|}{ TEMPERATURA $^{\mathbf{}} \mathbf{C}$} \\
\hline Características & $\mathbf{2 5}$ & $\mathbf{1 0 0}$ & $\mathbf{1 2 0}$ & $\mathbf{1 4 0}$ & $\mathbf{1 6 0}$ & $\mathbf{1 8 0}$ & $\mathbf{2 0 0}$ \\
\hline Gramatura (g/m $\mathbf{m}^{\mathbf{2}}$ & 93 & 97,4 & 100,6 & 102,8 & 104,6 & 105,8 & 108,4 \\
\hline Encolhimento trama (\%) & 0 & 0,5 & 1 & 1 & 1 & 2 & 4 \\
\hline Encolhimento urdume (\%) & 0 & 2 & 3 & 5 & 7 & 9 & 11 \\
\hline \% Along. Trama & 16 & 16 & 17 & 15 & 15 & 14 & 12 \\
\hline Tração trama (Kgf) & 36 & 36 & 35 & 37 & 40 & 42 & 38 \\
\hline \% Along. Urdume & 22 & 22 & 24 & 23 & 25 & 26 & 25 \\
\hline Tração Urdume (Kgf) & 49 & 49 & 49 & 49 & 50 & 52 & 47 \\
\hline
\end{tabular}

Observa-se na tabela 1 que com o aumento de temperatura de tratamento ocorre aumento da gramatura do tecido $\left(\mathrm{g} / \mathrm{m}^{2}\right)$, isto deve-se ao encolhimento sofrido pelo material, resultando desta forma o aumento de fios/cm. 


\section{AcoseQ \\ Congresso Brasileiro de Engenharia Química}

Nota-se também que no sentido de urdume ocorre maior encolhimento que no sentido de trama devida que as amostras são presas no suporte da estufa no sentido da trama, resultando tecido com menor tensão no sentido de urdume, propiciando maior facilidade de encolhimento neste sentido. Neste sentido, observa-se que a porcentagem de alongamento no sentido de urdume é maior, pois houve maior encolhimento.

A resistência a tração tende a aumentar com o aumento de temperatura até $180^{\circ} \mathrm{C}$, sugerindo aumento da cristalinidade, acima desta temperatura as propriedades de resistência a tração e \% de alongamento diminuem, resultando um material com baixa resistência e mais rígido, indicando inicio da degradação deste material.

Tabela 2. Resultados do ensaio de estabilidade dimensional de amostras de tecidos e poliéster tratadas termicamente.

\begin{tabular}{|c|c|c|c|c|c|c|c|c|}
\hline \multicolumn{3}{|c|}{$\begin{array}{l}\text { Temperatura de } \\
\text { Termofixação }{ }^{\circ} \mathrm{C}\end{array}$} & 25 & 100 & 140 & 160 & 180 & 200 \\
\hline \multicolumn{3}{|c|}{$\begin{array}{c}\text { Tempo de } \\
\text { Termofixação } \\
(\mathrm{seg})\end{array}$} & 0 & 120 & 120 & 120 & 120 & 120 \\
\hline \multirow{4}{*}{ 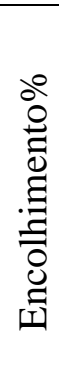 } & \multirow{4}{*}{ 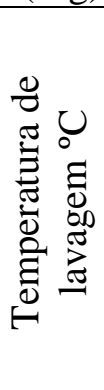 } & 25 & $\begin{array}{l}\mathrm{t}=1,0 \\
\mathrm{u}=1,0\end{array}$ & $\begin{array}{l}\mathrm{t}=0,0 \\
\mathrm{u}=0,0\end{array}$ & $\begin{array}{l}\mathrm{t}=0,0 \\
\mathrm{u}=0,0\end{array}$ & $\begin{array}{l}\mathrm{t}=0,0 \\
\mathrm{u}=0,0\end{array}$ & $\begin{array}{l}\mathrm{t}=0,0 \\
\mathrm{u}=0,0\end{array}$ & $\begin{array}{l}\mathrm{t}=0,0 \\
\mathrm{u}=0,0\end{array}$ \\
\hline & & 60 & $\begin{array}{l}\mathrm{t}=4,0 \\
\mathrm{u}=4,0\end{array}$ & $\begin{array}{l}\mathrm{t}=1,0 \\
\mathrm{u}=1,0\end{array}$ & $\begin{array}{l}\mathrm{t}=1,0 \\
\mathrm{u}=1^{\prime}, 0\end{array}$ & $\begin{array}{l}\mathrm{t}=1,0 \\
\mathrm{u}=1,0\end{array}$ & $\begin{array}{l}\mathrm{t}=1,0 \\
\mathrm{u}=1,0\end{array}$ & $\begin{array}{l}\mathrm{t}=0,0 \\
\mathrm{u}=0,0\end{array}$ \\
\hline & & 80 & $\begin{array}{l}\mathrm{t}=10,0 \\
\mathrm{u}=12,0\end{array}$ & $\begin{array}{l}\mathrm{t}=3,0 \\
\mathrm{u}=3,0\end{array}$ & $\begin{array}{l}\mathrm{t}=3,0 \\
\mathrm{u}=3,0\end{array}$ & $\begin{array}{l}\mathrm{t}=0,0 \\
\mathrm{u}=0,0\end{array}$ & $\begin{array}{l}\mathrm{t}=0,0 \\
\mathrm{u}=0,0\end{array}$ & $\begin{array}{l}\mathrm{t}=0,0 \\
\mathrm{u}=0,0\end{array}$ \\
\hline & & 96 & $\begin{array}{l}\mathrm{t}=14,0 \\
\mathrm{u}=20,0\end{array}$ & $\begin{array}{l}\mathrm{t}=4,0 \\
\mathrm{u}=4,0\end{array}$ & $\begin{array}{l}\mathrm{t}=4,0 \\
\mathrm{u}=4,0\end{array}$ & $\begin{array}{l}\mathrm{t}=0,0 \\
\mathrm{u}=0,0\end{array}$ & $\begin{array}{l}\mathrm{t}=0,0 \\
\mathrm{u}=0,0\end{array}$ & $\begin{array}{l}\mathrm{t}=0,0 \\
\mathrm{u}=0,0\end{array}$ \\
\hline
\end{tabular}

A tabela 2 mostra que tecidos termofixados até $140^{\circ} \mathrm{C}$ apresentam encolhimento quando submetidos a ensaios de estabilidade dimensional, ou seja, sofrem encolhimento quando tratados em banhos aquecidos, causando deformação no tecido. Tecidos tratados a partir de $160^{\circ} \mathrm{C}$ não sofrem mais encolhimento quando submetidos a tratamento em banhos aquecidos, indicando desta forma que já obtiveram a estabilidade dimensional 


\subsection{Tecido de poliamida:}

Tabela 3- Resultados das propriedades dos tecidos de poliamida tratados termicamente nas temperaturas de $100,120,140,160,180$ e $200^{\circ} \mathrm{C}$ e de tecidos não tratados $\left(25^{\circ} \mathrm{C}\right)$.

\begin{tabular}{|c|c|c|c|c|c|c|c|}
\hline \multirow[b]{2}{*}{ Características } & \multicolumn{7}{|c|}{ TEMPERATURA $^{\circ} \mathrm{C}$} \\
\hline & & & & & & & \\
\hline & & & & & & & \\
\hline Gramatura $\left(\mathrm{g} / \mathrm{m}^{2}\right)$ & 74,7 & 77,1 & 78,4 & 78,8 & 79.2 & 82,0 & 82,9 \\
\hline Encolhimento trama (\%) & 0 & 0 & 0,5 & 0,5 & 0,5 & 1 & 3 \\
\hline Encolhimento urdume $\quad(\%)$ & 0 & 0,5 & 0,5 & 1,0 & 1,5 & 5,0 & 7,0 \\
\hline \% Along. Trama & 60,8 & 60,5 & 62,8 & 64.4 & 67,3 & 59,3 & 58,8 \\
\hline Tração trama ( Kgf) & 43,2 & 49,5 & 52,8 & 55,6 & 62,2 & 60,2 & 57,2 \\
\hline \% Along. Urdume & 41,9 & 37,2 & 41,1 & 42,3 & 49,1 & 46,8 & 35,6 \\
\hline Tração Urdume (Kgf) & 61,9 & 61,7 & 63,9 & 64,6 & 63,7 & 65,5 & 63,6 \\
\hline
\end{tabular}

A tabela 3 indica aumento da gramatura de tecidos de poliamida em relação ao aumento de temperatura de termofixação. Isto ocorre porque há encolhimento do tecido, que por consequiência aumenta-se a quantidade de fios por unidade de área, resultando o acréscimo na massa do tecido. A resistência a tração e alongamento tendem a aumentar tanto no sentido de urdume (comprimento) como no sentido de trama (largura) até $160^{\circ} \mathrm{C}$, pois da mesma forma que na gramatura, com o encolhimento aumenta-se a elasticidade do material e a resistência porque existe maior quantidade de fios nas amostras submetidas aos ensaios de resistência a tração. Nas temperaturas superiores a $160^{\circ} \mathrm{C}$, estas propriedades diminuem, indicando que o material apresenta maior rigidez (diminuição do alongamento) e degradação do tecido (diminuição da resistência a tração. 


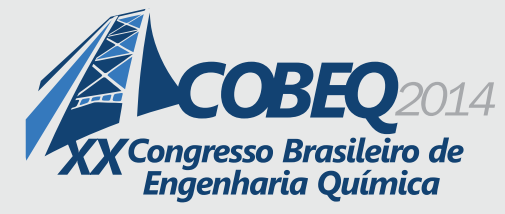

19 a 22 de outubro de 2014

Florianópolis/SC

Tabela 4: Resultados do ensaio de estabilidade dimensional de tecidos de poliamida tratados termicamente e não tratados

\begin{tabular}{|c|c|c|c|c|c|c|c|c|c|}
\hline \multicolumn{3}{|c|}{$\begin{array}{c}\text { Temperatura de } \\
\text { Termofixação }{ }^{\circ} \mathrm{C}\end{array}$} & \begin{tabular}{|c} 
Sem \\
tratamento
\end{tabular} & 100 & 120 & 140 & 160 & 180 & 200 \\
\hline \multicolumn{3}{|c|}{$\begin{array}{c}\text { Tempo de } \\
\text { Termofixação (seg) }\end{array}$} & 0 & 120 & 120 & 120 & 120 & 120 & 120 \\
\hline \multirow{4}{*}{ 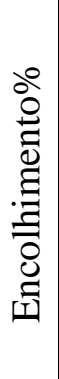 } & \multirow{4}{*}{ 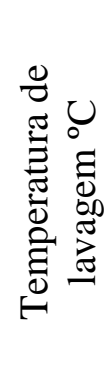 } & 40 & $\begin{array}{l}\mathrm{t}=0,0 \\
\mathrm{u}=0,0\end{array}$ & $\begin{aligned} \mathrm{t} & =0,0 \\
\mathrm{u} & =0,0\end{aligned}$ & $\begin{array}{l}\mathrm{t}=0,0 \\
\mathrm{u}=0,0\end{array}$ & $\begin{array}{l}\mathrm{t}=0,0 \\
\mathrm{u}=0,0\end{array}$ & $\begin{aligned} \mathrm{t} & =0,0 \\
\mathrm{u} & =0,0\end{aligned}$ & $\begin{array}{l}\mathrm{t}=0,0 \\
\mathrm{u}=0,0\end{array}$ & $\begin{array}{l}\mathrm{t}=0,0 \\
\mathrm{u}=0,0\end{array}$ \\
\hline & & 60 & $\begin{array}{l}\mathrm{t}=2,0 \\
\mathrm{u}=0,0\end{array}$ & $\begin{aligned} \mathrm{t} & =1,0 \\
\mathrm{u} & =0,0\end{aligned}$ & $\begin{array}{l}\mathrm{t}=0,5 \\
\mathrm{u}=0,0\end{array}$ & $\begin{array}{l}\mathrm{t}=0,0 \\
\mathrm{u}=0,0\end{array}$ & $\begin{aligned} \mathrm{t} & =0,0 \\
\mathrm{u} & =0,0\end{aligned}$ & $\begin{array}{l}\mathrm{t}=0,0 \\
\mathrm{u}=0,0\end{array}$ & $\begin{array}{l}\mathrm{t}=0,0 \\
\mathrm{u}=0,0\end{array}$ \\
\hline & & 80 & $\begin{array}{l}\mathrm{t}=5,0 \\
\mathrm{u}=0,0\end{array}$ & $\begin{aligned} \mathrm{t} & =2,0 \\
\mathrm{u} & =0,0\end{aligned}$ & $\begin{array}{l}\mathrm{t}=1,0 \\
\mathrm{u}=0,0\end{array}$ & $\begin{array}{l}\mathrm{t}=0,0 \\
\mathrm{u}=0,0\end{array}$ & $\begin{aligned} \mathrm{t} & =0,0 \\
\mathrm{u} & =0,0\end{aligned}$ & $\begin{array}{l}\mathrm{t}=0,0 \\
\mathrm{u}=0,0\end{array}$ & $\begin{array}{l}\mathrm{t}=0,0 \\
\mathrm{u}=0,0\end{array}$ \\
\hline & & 96 & $\begin{array}{l}\mathrm{t}=5,0 \\
\mathrm{u}=0,0\end{array}$ & $\begin{array}{l}\mathrm{t}=3,0 \\
\mathrm{u}=0,0\end{array}$ & $\begin{array}{l}\mathrm{t}=2,0 \\
\mathrm{u}=0,0\end{array}$ & $\begin{array}{l}\mathrm{t}=0,0 \\
\mathrm{u}=0,0\end{array}$ & $\begin{array}{l}\mathrm{t}=0,0 \\
\mathrm{u}=0,0\end{array}$ & $\begin{array}{l}\mathrm{t}=0,0 \\
\mathrm{u}=0,0\end{array}$ & $\begin{array}{l}\mathrm{t}=0,0 \\
\mathrm{u}=0,0\end{array}$ \\
\hline
\end{tabular}

A tabela 4 mostra os resultados dos ensaios de estabilidade dimensional de amostras de tecidos de poliamida tratadas termicamente. Observa-se que os tecidos tratados até $120^{\circ}$ apresentam encolhimento quando submetidos a tratamentos em banhos aquecidos, ou seja, não atingiram a estabilidade dimensional. Esta estabilidade ocorre a $140^{\circ} \mathrm{C}$, indicando que nesta temperatura o tecido não sofrerá mais encolhimento em banhos aquecidos até a temperatura de $96^{\circ} \mathrm{C}$. Nos processos após ao tratamento térmico (termofixação), como por exemplo o tingimento que é realizado a temperatura de ebulição o tecido não sofrerá alterações dimensionais (sem encolhimento).

\subsection{Tingimentos de poliéster e poliamida tratados e não tratados termicamente:}

Observa-se que, com o aumento de temperatura ocorre menor absorção de corante, indicando que houve alterações nas estruturas das fibras com o tratamento térmico, principalmente em relação a sua cristalinidade, que aumenta com a temperatura elevada, a qual proporciona maior dificuldade de absorção de corante. Neste sentido, tratando-se os tecidos em diferentes temperaturas podem ocorrer diferentes tonalidades do tingimento, ou seja, produzindo tecidos com cores diferentes ao padrão desejado, o que não é bom para qualidade final do tecido. Assim é importante na preparação destes tecidos antes do tingimento que se mantenha constante a temperatura do tratamento térmico. 


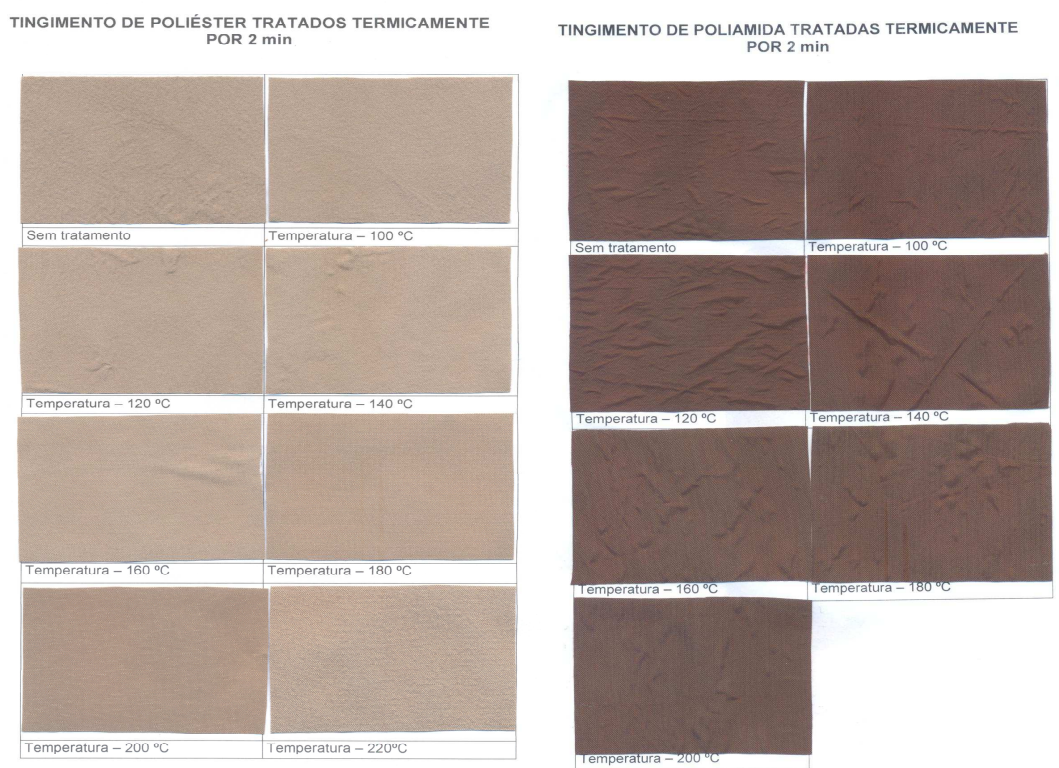

Figura 1: Tingimento de tecidos de poliéster e poliamida após tratamento térmico.

\section{CONCLUSÕES:}

- A temperatura de $160^{\circ} \mathrm{C}$ de tratamento em poliéster e $140^{\circ} \mathrm{C}$ são o suficiente para se obter boa estabilidade dimensional,

- A resistência à tração e alongamento aumenta com o aumento de temperatura até $180^{\circ}$ para poliéster e $140^{\circ}$ para poliamida, acima destas temperaturas estas propriedades diminuem, indicando que o inicio da degradação destes materiais;

- O tratamento térmico influi na absorção de corante: neste tratamento ocorre aumento das regiões cristalinas das fibras sintéticas, com isso dificultando a penetração do corante, resultando cores mais claras com o aumento da temperatura;

\section{REFERÊNCIAS BIBLIOGRÁFICAS:}

1. BERNARD, P. C., (1983). Textiles Fibers to Fabrics, 6 ${ }^{\text {a }}$ edição. Mc Graw International Editions. New York.

2. BILLMEYER, F.W. Jr. "Textbook of Polymer Science", 2 th . Edition, New York, John Wiley \& Sons. , (1984),

3. MENACHEN, L., PRESTON, J., (1993). High Technology Fibers. Part A, Volume 3, Marcel Dekker, New York.

4. ODIAN, G. Principles of Polymerization. 3 ed. McGraw-Hill Inc., New York, USA.1991. $475 \mathrm{p}$.

5. VASQUEZ, N.R.; SAKELLÁRIOS, A. S. Foto e tratamento térmico de polímeros. In: $1^{\circ}$ SEMINÁRIO TÉCNICO SOBRE POLÍMEROS. Salvador. BA. Anais.1980. p.187 a 205. 